\title{
Anomalías de la experiencia subjetiva en psicosis: Concepto y validación empírica del modelo de los Síntomas Básicos
}

\author{
Raül Vilagrà Ruiz, ${ }^{1}$ Neus Barrantes-Vidal ${ }^{1}$
}

Actualización por temas

\section{ABSTRACT}

Introduction

Research, diagnosis and treatment of psychotic spectrum disorders have been traditionally dominated by an objectivist approach to their understanding, being primarily focused on positive and negative symptoms. The value of this approach goes without question, but it also involves considerable and widely known limitations. From a complementary perspective, there is a longstanding and promising phenomenological tradition in which the subjective experience of the patient's symptom becomes crucial. The focus on the anomalies of subjective experience, or the Basic Symptom concept specifically, has gained much momentum in the context of early detection of psychosis and schizophrenia.

\section{Objective}

This review presents the phenomenological approach to the anomalies of subjective experience and the Basic Symptoms model and its empirical validation process in the field of early detection of psychosis.

\section{Method}

The scientific literature was collected from PubMed Central ${ }^{\circledR}$ and PsycINFO ${ }^{\circledR}$ databases and books from authors of reference.

\section{Results}

In the last two decades there has been a growing scientific interest in this approach with very promising results.

\section{Discussion and conclusion}

The most prominent model from an empirical standpoint is the Basic Symptoms approach, although recently the disturbances of the flow of consciousness or self disorders have achieved great relevance as well.

It has been found that the anomalies of subjective experience could delimitate a risk profile that precedes that defined by attenuated psychotic symptoms. Therefore, this approach is a highly valuable complement in the early detection and intervention of psychosis strategies.

Key words: Psychosis, schizophrenia, Basic Symptoms, subjective experience, prodrome, risk.

\section{RESUMEN}

Introducción

Tradicionalmente, en la investigación, el diagnóstico y el tratamiento de los trastornos del espectro psicótico ha imperado un modelo de comprensión objetivista, centrado principalmente en los síntomas positivos y negativos. Aunque es innegable el valor de esta aproximación, implica considerables limitaciones ampliamente conocidas. De forma complementaria, existe una larga y prometedora tradición fenomenológica en la cual la experiencia subjetiva del síntoma del paciente adquiere una importancia fundamental. La aproximación de las anomalías de la experiencia subjetiva 0 , específicamente, de los Síntomas Básicos ha adquirido mucha fuerza dentro del contexto de detección precoz de psicosis y esquizofrenia.

\section{Objetivo}

Esta revisión expone la aproximación fenomenológica de las anomalías de la experiencia subjetiva y se define detalladamente el modelo de los Síntomas Básicos, así como su proceso de validación empírica en el campo de detección precoz de psicosis.

\section{Método}

Las bases de datos consultadas han sido PubMed Centra ${ }^{\circledR}$ y PsycINFO ${ }^{\circledR}$, así como libros de autores de referencia.

\section{Resultados}

En las dos últimas décadas ha habido un creciente interés científico sobre esta orientación con resultados muy prometedores.

\section{Discusión y conclusión}

El modelo más destacado a nivel empírico es el de los Síntomas Básicos, aunque recientemente también han ganado gran relevancia las alteraciones del flujo de la consciencia o del self.

Se ha comprobado que las anomalías de la experiencia subjetiva consiguen delimitar un perfil de riesgo de psicosis más temprano que los síntomas psicóticos atenuados. Por tanto, son un complemento altamente válido en las estrategias de detección e intervención temprana de psicosis.

Palabras clave: Psicosis, esquizofrenia, Síntomas Básicos, experiencia subjetiva, pródromo, riesgo. 


\section{INTRODUCCIÓN}

Un aspecto común de la investigación, diagnóstico y tratamiento de las esquizofrenias es que se han focalizado principalmente en los llamados síntomas positivos y negativos. Este énfasis en la vertiente clínica-objetiva de las manifestaciones psicóticas se explica en gran parte por la preeminencia del paradigma epistemológico conductista, con la consiguiente búsqueda de una medición fiable de un fenómeno, ${ }^{1}$ por la popularidad de los criterios diagnósticos Schneiderianos, ${ }^{2}$ y por la dificultad de la psicopatología fenomenológica en traducir sus conocimientos y hallazgos en términos lo suficientemente operativos para ser utilizados en la investigación y el diagnóstico psicopatológicos. ${ }^{3}$ Lo anterior ha implicado que en las clasificaciones diagnósticas actuales y en algunos grandes estudios prospectivos de detección precoz de psicosis no se haya incluido la visión fenomenológica de las anomalías de la experiencia subjetiva, pese a que hayan sido consideradas frecuentemente por la psiquiatría clásica y descritas detalladamente en los trastornos esquizofrénicos por la psicopatología fenomenológica.

Después de más de dos décadas de investigación intensiva en detección precoz de psicosis, se está haciendo cada vez más evidente la necesidad de recuperar la perspectiva fenomenológica en el estudio de las manifestaciones psicóticas, tradicionalmente demasiado limitada por una concepción categorial que no favorece su refinamiento, o que directamente impide su investigación y tratamiento. ${ }^{4}$ Dicha perspectiva implica observar y describir cuidadosamente un fenómeno cómo éste se manifiesta, dando un valor preponderante a la experiencia que el paciente describe como propia de su mundo subjetivo. Con ello se pretende recuperar la perspectiva de análisis narrativo o en primera persona del sufrimiento psicopatológico. ${ }^{5}$

En esencia, el concepto de las Anomalías de la Experiencia Subjetiva (AES) estaría principalmente representado por el modelo fenomenológico de los "Síntomas básicos de Huber". Los Síntomas Básicos (SB) se definen como perturbaciones sutiles, subclínicas e inespecíficas experimentadas a nivel subjetivo en la motivación, el afecto, la cognición, la percepción, el habla, la tolerancia al estrés, la percepción corporal, la actividad motora y las funciones centrales vegetativas.6-10 Dentro de las AES, e íntimamente ligada al modelo de los SB, también se incluiría la aproximación clínica y filosófica de las alteraciones del self (self-disorders; basic selfdisturbances), la cual ha experimentado un gran desarrollo conceptual y empírico en los últimos años. Aunque ambas aproximaciones comparten en sus protocolos de evaluación muchas descripciones de síntomas, las alteraciones del self se focalizarían de forma más específica en las llamadas experiencias anómalas de la autoconciencia, definidas como una experiencia generalizada o frecuentemente recurrente en la cual la perspectiva de sí mismo, como sujeto de la experiencia o la acción, está de alguna forma distorsionada. ${ }^{1,4, *}$

* Para una revisión teórica en castellano de las anomalías del self, véase Pérez-Álvarez, García-Montes y Sass. ${ }^{11}$
En la presente revisión se define al modelo fenomenológico de los $\mathrm{SB}$, se expone específicamente el proceso de validación y evaluación empírica de los SB, y se concluye con una integración comprensiva de las aportaciones más importantes de este modelo y, por extensión, de la aproximación de las AES dentro del campo de la detección precoz de psicosis.

\section{EL MODELO DE LOS SÍNTOMAS BÁSICOS}

Los SB fueron descritos por primera vez por Gerd Huber, alumno de Kurt Schneider en el Hospital Universitario de Heidelberg en la década de los 1950. Actualmente, este modelo ha sido continuado y validado a nivel empírico-predictivo por Frauke Schultze-Lutter, Joachim Klosterkötter y Stephan Ruhrmann, entre otros. El concepto de SB se podría resumir como sigue según esta traducción de Jimeno-Bulnes, Jimeno-Valdés y Vargas (1996) ${ }^{12}$ de un texto original en alemán de Huber:

"Síntomas psicóticos recogidos en esquizofrénicos como vivencias
subjetivas primarias que constituyen la base de los complejos sín-
tomas psicóticos finales, y que puede suponerse se encuentran más
próximos al sustrato neurobiológico. Son, pues, síntomas deficitarios,
con carácter de queja por parte de los pacientes, que desde el punto
de vista fenomenológico coinciden ampliamente con síntomas pre-
monitorios y prodrómicos prepsicóticos, y que en los estadios básicos
reversibles y en el síndrome defectual puro irreversible son percibidos
y expresados como deficiencias, carencias o trastornos."

En otras palabras, los SB son la expresión psicopatológica más inmediata de una supuesta susceptibilidad o alteración orgánica primordial, de ahí el término "básico"; sustentan en forma de déficits o alteraciones básicas los posibles síntomas psicóticos productivos subsiguientes; y pueden aparecer de forma inespecífica en cualquier estadio del trastorno esquizofrénico (prepsicótico-psicótico-postpsicótico), es decir, son parte integral de todo el proceso esquizofrénico. En la literatura se pueden encontrar distintos términos para designar los SB, como por ejemplo las disfunciones cognitivas subjetivas, los síntomas subclínicos o las experiencias subjetivas. ${ }^{2}$ Las dos características definitorias de los SB se refieren a su cualidad subjetiva, tratándose de síntomas o experiencias subjetivas que el paciente vive como propias de su mundo interno o privado, y a su sutileza o naturaleza subclínica, experimentándose de forma frecuente con extrañeza o duda, aunque siempre reconociendo estos déficits como propios de la realidad mental de quien los experimenta. Lo anterior implica que es la propia persona afectada quien determina si el fenómeno existe o no. Los SB no son exclusivos de la esquizofrenia, pudiendo ser encontrados también en otros trastornos del espectro psicótico-afectivo. ${ }^{10,13}$

En suma, el modelo de los SB concibió una nueva visión evolutiva y psicopatológica de la esquizofrenia que sometió a revisión la clásica doctrina de la heterogeneidad de este trastorno, al plantear la existencia de una sintomatología 
Cuadro 1. Síntomas Básicos Cognitivo-Perceptivos (COPER)

Presencia de al menos uno de los siguientes diez síntomas básicos con una puntuación en SPI-A/SPI-CY $\geq 3$ dentro de los últimos tres meses y con la primera ocurrencia $\geq$ a 12 meses

Interferencia del pensamiento

Perseveración del pensamiento

Presión del pensamiento

Bloqueo del pensamiento

Alteración del habla receptiva

Disminución de la habilidad para discriminar entre ideas y percepción, fantasía y recuerdos verdaderos

Ideas de referencia inestables

Desrealización

Alteraciones de la percepción visual

Alteraciones de la percepción acústica

fundamental pero sutil, esto es, básica, presente en todo el proceso esquizofrénico. Esta nueva aproximación enriquece el estudio y diagnóstico de las fases iniciales, intermedias y tardías del trastorno, y fomenta la implantación de nuevas intervenciones preventivas, terapéuticas y rehabilitadoras. ${ }^{6,7,14}$

\section{Validación empírica de los Síntomas Básicos}

La fenomenología de los SB ha sido ampliamente analizada en numerosas investigaciones prospectivas de detección precoz de psicosis durante la última década, culminando con la elaboración de dos criterios de riesgo de psicosis: los SB cognitivo-perceptivos (At-Risk Criterion Cognitive-Perceptive Basic Symptom, COPER) y las alteraciones cognitivas de alto riesgo (High-risk Criterion Cognitive Disturbances, COGDIS) (cuadros 1 y 2). Estos criterios derivaron del estudio prospectivo Cologne Early Recognition (CER). ${ }^{15}$ Durante una media de 9.6 años se investigaron mediante la Bonn Scale for the Assessment of Basic Symptoms (BSABS) ${ }^{16,17} 160$ pacientes que presentaban alteraciones afines al pródromo psicótico pero que nunca habían sufrido un episodio psicótico franco. Tras un análisis de todos los ítems de la BSABS, se encontró un subgrupo de alteraciones cognitivas y perceptivas que re-

\section{Cuadro 2. Alteraciones Cognitivas de Alto Riesgo (COGDIS)}

Presencia de al menos dos de los siguientes nueve síntomas básicos con una puntuación en SPI-A/SPI-CY $\geq 3$ dentro de los últimos tres meses

Incapacidad para dividir la atención

Interferencia del pensamiento

Presión del pensamiento

Bloqueo del pensamiento

Alteración del habla receptiva

Alteración del habla expresiva

Ideas de referencia inestables

Alteraciones del pensamiento abstracto

Captación de la atención por detalles del campo visual sultaron ser altamente predictivas de psicosis, elaborándose el criterio COPER. Estos SB mostraron cada uno de ellos un valor predictivo alto [área bajo la curva (AUC) $\geq .70$ ] y estuvieron presentes en al menos un $25 \%$ de los sujetos que transitaron a psicosis. Como punto de corte para cumplir el criterio, se estableció estadísticamente la presencia de al menos uno de los diez SB, resultando para el criterio COPER una sensibilidad final del $87 \%$, una especificidad del $54 \%$ y un poder predictivo positivo del $65 \%$.

El criterio COGDIS derivó de un nuevo análisis de los datos del estudio CER, hallando una nueva combinación de SB cuya agrupación demostró ser la más predictiva en comparación con otros siete grupos de síntomas. ${ }^{18}$ Se estableció como punto de corte la presencia de al menos dos de los nueve SB, resultando para el criterio COGDIS una sensibilidad final del $67 \%$, una especificidad del $83 \%$ y un poder predictivo positivo del $79 \%$. Como puede apreciarse en los cuadros, el criterio COGDIS está parcialmente solapado con el criterio COPER al compartir con este cinco de los nueve SB. Si bien ambos criterios de riesgo ofrecen una precisión predictiva similar (COPER: AUC=0.83; COGDIS: AUC =0.82), se encontró que el criterio COGDIS delineaba un riesgo de psicosis ligeramente más inminente al encontrarse tasas de transición a psicosis algo más elevadas en el grupo COGDIS en cada uno de los cuatro años de seguimiento de la muestra. ${ }^{18}$

La gravedad de los SB y el diagnóstico de los criterios de riesgo se pueden definir según distintos criterios cualitativos y cuantitativos mediante el Instrumento de Vulnerabilidad a la Esquizofrenia, versión para adultos (Schizophrenia Proneness Instrument, Adult Version, SPI-A), ${ }^{19}$ disponible también en castellano, y versión para niños y adolescentes (Schizophrenia Proneness Instrument, Child and Youth Version, SPI-CY), ${ }^{20}$ los cuales serán expuestos más adelante.

Los valiosos datos del estudio CER, por tanto, supusieron un paso significativo en la demostración de la validez predictiva de psicosis de muchos de los SB. En consecuencia, otro estudio enmarcado dentro del European Early Detection and Intervention Centre (FETZ) ${ }^{21}$ reevaluó la capacidad predictiva de los criterios COPER/COGDIS en una muestra de 146 sujetos con síntomas prodrómicos evaluados con la SPI-A y la Positive and Negative Syndrome Scale (PANSS). ${ }^{22}$ Los resultados hallaron que 124 sujetos cumplieron criterios COPER y COGDIS y 22 únicamente el criterio COPER. Se encontró que los sujetos que cumplían criterios COGDIS presentaban una intensidad sintomatológica significativamente mayor en todas las subescalas de la SPI-A y PANSS, salvo en la dimensión de síntomas negativos de esta última. Aunque estos hallazgos confirmaron la buena validez predictiva de los SB, no apoyaron la noción de que los criterios COPER per se delinearan un riesgo de psicosis menos inminente que los COGDIS al encontrarse tasas de transición a psicosis similares en ambos criterios de riesgo. ${ }^{9}$ 
Vilagrà Ruiz y Barrantes-Vidal

\section{Evaluación de los Síntomas Básicos}

Aunque la elaboración y diagnóstico de los criterios de riesgo COPER y COGDIS es relativamente reciente, anteriormente ya existía una larga investigación sobre la especificad de los SB en relación a la psicosis. El principal instrumento de evaluación de los SB es la ya mencionada Escala de Bonn para la evaluación de síntomas básicos Bonn Scale for the Assessment of Basic Symptoms, (BSABS). ${ }^{16,17}$ Se trata de una entrevista clínica semiestructurada, de administración heteroaplicada y formato de respuesta binario. En ella se recogen 98 ítems o SB definidos operacionalmente mediante preguntas y ejemplos representativos del síntoma tomados de la experiencia de otros pacientes que facilitan la evaluación. De forma orientativa, la BSABS se estructura en cinco categorías principales de síntomas: deficiencias dinámicas, cognición y pensamiento, percepción, alteraciones motoras y cenestesias y alteraciones centrales vegetativas. Aunque este es el instrumento más completo y referencial para la evaluación de los SB, requiere de mucho tiempo de aplicación y entrenamiento previo, lo que puede dificultar su uso clínico y de investigación.

A partir de los datos del estudio CER se construyó un nuevo instrumento basado en la BSABS, el ya mencionado Instrumento de Vulnerabilidad a la esquizofrenia, versión para adultos (SPI-A). ${ }^{19}$ La SPI-A fue diseñada empíricamente, lo que garantiza la inclusión de todos aquellos SB que han demostrado en estudios prospectivos alta especificidad para psicosis. Tras analizar dos tipos de muestras, una de 160 sujetos prodrómicos y otra de 346 sujetos con diagnóstico de esquizofrenia, se generaron seis dimensiones o subescalas de SB altamente robustas y fiables en ambas muestras de pacientes: alteraciones afectivas-dinámicas (deterioro de la tolerancia al estrés, cambios en el estado de ánimo, etc.), dificultades cognitivas-atencionales (alta distracción por todo tipo de estímulos, dificultades de memoria a corto plazo y concentración, etc.), alteraciones cognitivas (interferencia del pensamiento, bloqueo del pensamiento, etc.), alteraciones en la vivencia del Yo y del entorno (aumento de la reactividad emocional, ideas de referencia inestables, etc.), alteraciones de la percepción corporal (varios tipos de sensaciones corporales inusuales), y por último, alteraciones de la percepción (fotopsia, micropsia, etc.). Para una descripción detallada de las escalas, véase Schultze-Lutter et al. ${ }^{19,23}$ La dimensión que mostró más precisión diagnóstica fue la de alteraciones cognitivas. La estabilidad de la estructura dimensional hallada a lo largo de los diferentes estadios del trastorno llevó a la conclusión de que estas seis dimensiones eran inherentes a la esquizofrenia. ${ }^{24}$

De igual forma que la BSABS, la SPI-A también fue concebida como una entrevista semi-estructurada, pero el formato de respuesta dejó de ser binario para convertirse en dimensional según una escala de gravedad de 6 puntos. Para cada SB se establecen unos criterios de codificación se- gún su relevancia, siendo los más importantes la frecuencia y la gravedad.

Un aspecto común y restrictivo de los criterios de riesgo de psicosis actuales es que se han desarrollado sólo o predominantemente en muestras de adultos, ${ }^{25}$ por consiguiente, hasta ahora el diseño de los instrumentos de detección precoz de psicosis ha sido basado en este perfil poblacional. Además, son escasas las investigaciones que estudian prevención e intervención de las psicosis de inicio precoz en población infanto-juvenil ( $<18$ años). ${ }^{26,27}$ De modo relevante, el German-Austrian-Swiss multicenter study (VESPA) ${ }^{28}$ encontró que la prevalencia de los SB en una muestra de adolescentes de la población general es alta (30.2\%), aumentando a $81 \%$ en la muestra de adolescentes con trastornos psiquiátricos no psicóticos y hasta el $96.5 \%$ en los casos con psicosis de inicio precoz. Todo lo anterior, más la ausencia de una herramienta clínica de riesgo de psicosis diseñada para este grupo de edad, puede suponer que se desatiendan importantes aspectos diferenciales de la población infantil y adolescente.

Para subsanar esta carencia se desarrolló la Schizophrenia Proneness Instrument, Child and Youth Version (SPI-CY), ${ }^{20}$ instrumento específicamente diseñado para este grupo de edad según el modelo de los SB. Inicialmente, se intentó replicar la misma estructura dimensional encontrada en la SPI-A en un muestra de 32 sujetos con psicosis de inicio precoz (<18 años), pero los datos no apoyaron dicha estructura. En consecuencia, se llevaron a cabo nuevos análisis que revelaron una nueva estructura de cuatro dimensiones que engloba un total de 49 ítems de la BSABS. ${ }^{29}$ Éstas son: adinamia, alteraciones perceptivas, alteraciones cognitivas y neuroticismo. En comparación con la versión para adultos, destacan dos dimensiones que son específicas de la SPI-CY: la dimensión adinamia, la cual cubre síntomas presentes en las dimensiones de alteraciones afectivas-dinámicas y dificultades cognitivas-atencionales de la versión para adultos, más otros síntomas referentes a falta de energía, persistencia, motivación, episodios depresivos y la dimensión neuroticismo, que contiene síntomas referentes a deseo reducido para interacciones sociales, aumento de la reactividad emocional, irritabilidad, fenómenos fóbicos, entre otros. ${ }^{20,23}$ En cuanto a las propiedades psicométricas de la SPI-CY, hasta la fecha se ha encontrado según un estudio que ha comparado tres muestras de niños y adolescentes (23 sujetos con riesgo de psicosis; 22 sujetos controles con problemas no psicóticos; y 19 sujetos de la población general), que las subescalas de la SPI-CY poseen una alta validez discriminativa entre grupos, destacando especialmente la dimensión adinamia. ${ }^{30}$ En suma, la SPI-CY representa el día de hoy la única herramienta de detección precoz de psicosis diseñada para ser usada en población infanto-juvenil, aunque requiere todavía de más estudios prospectivos que la validen. ${ }^{23,30,31}$ 


\section{LAS ANOMALÍAS DE LA EXPERIENCIA SUBJETIVA Y EL SÍNDROME DE RIESGO PRECOZ DE PSICOSIS}

Básicamente, se pueden resumir dos grandes hitos en el campo de detección precoz de psicosis: la definición de un síndrome o estado de alto riesgo de psicosis según unos criterios clínicos y funcionales más o menos consensuados, y el desarrollo paralelo de herramientas clínicas para evaluar dicho estado de riesgo. El término "Ultra High Risk" (UHR), ${ }^{32}$ proveniente de la escuela australiana y muy extendido en Europa, fue ampliamente aceptado como un conjunto de criterios de riesgo inminente de psicosis a finales de los 1990. No obstante, con frecuencia se ha intercambiado este término con el de "At Risk Mental States" (ARMS), ${ }^{33}$ concepto más bien definitorio y aglutinador de un posible estado prodrómico, o con el "Clinical High Risk" (CHR), ${ }^{34}$ de la escuela norteamericana.

Principalmente, los criterios UHR combinan tres indicadores de riesgo: 1. Riesgo familiar (de primer o segundo grado) y/o trastorno de personalidad esquizotípica, junto con un deterioro funcional o psicosocial $\geq 30 \% ; 2$. Síntomas psicóticos atenuados Attenuated Positive Symptoms (APS) y 3. Síntomas psicóticos breves limitados intermitentes Brief Limited Intermitent Psychotic Symptoms (BLIPS). En cuanto a los intrumentos de evaluación de estos criterios, la Comprehensive Assessment of At-Risk Mental States (CAARMS) ${ }^{35}$ y la Structural Interview for Psychosis Syndromes (SIPS) junto con la Scale of Prodromal Symptoms (SOPS), ${ }^{36}$ conforman la evaluación e investigación tradicional de los UHR y CHR respectivamente.

Pese a la validez predictiva del estado UHR, se ha demostrado la existencia de síntomas tempranos y altamente inespecíficos que también forman parte del pródromo, y que son difícilmente distinguibles de las alteraciones del ánimo, motivación, sociabilidad o concentración típicos de los episodios depresivos. ${ }^{37}$ En este sentido, en un estudio retrospectivo en pacientes con primer episodio psicótico, ${ }^{38}$ se estableció una secuencia sintomática temporal que se iniciaba con sintomatología inespecífica, la cual ocurría como media 8.2 años antes del primer brote psicótico, seguida por SB de tipo cognitivoperceptivo junto con síntomas psicóticos atenuados (APS), con una media de 3.3 años antes del primer episodio psicótico.

Estos datos reflejarían una hipotética evolución psicopatológica secuencial (síntomas inespecíficos $\rightarrow \mathrm{SB} / \mathrm{APS} \rightarrow$ síntomas psicóticos) en la cual las alteraciones subjetivas relativas al procesamiento de información (SB cognitivos) precederían con mucho la emergencia de síntomas específicamente psicóticos, apoyando así la noción de que el enfoque fenomenológico de los SB sería complementario al UHR en la detección precoz de psicosis. ${ }^{24}$ En esta línea, otro estudio encontró que la inclusión de los SB (COPER) junto con los UHR definía una muestra más homogénea a nivel de deterioro clínico y cognitivo, lo cual aumenta la sensibilidad para predecir qué individuos desarrollarán psicosis. ${ }^{39}$ Esta noción de complementariedad se ve apoyada por el hecho de que el criterio UHR es especialmente válido para detectar individuos con riesgo inminente de psicosis según estudios prospectivos, no sólo al año de la evaluación basal, sino también a los seis meses, y que los criterios según SB consiguen detectar síntomas igualmente predictivos de modo más temprano al mantener unos porcentajes de transición a psicosis más bajos y estables más allá de los primeros doce meses, fenómeno que es consistente con la presentación insidiosa de los SB antes del primer brote psicótico.

Integrando los hallazgos referentes a la validez predictiva de los criterios COPER/COGDIS y UHR, junto con la noción de una secuencia sintomática subyacente en las psicosis, se ha sugerido un modelo de riesgo clínico estructurado en fases que permite delinear tres grupos de riesgo: ${ }^{37} 1$. El llamado "Estado de Riesgo de Psicosis Temprano" Early At-Risk of Psychosis State (ERPS), formado por la presencia de $\mathrm{SB}$ de riesgo (COPER) y/o un estado de riesgo biológico con deterioro funcional de al menos $\geq 30 \%$, definido bien por la existencia de familiares de primer grado con diagnóstico de esquizofrenia o un trastorno del espectro esquizofrénico, o bien por historia de complicaciones obstétricas. 2. Estado de riesgo de psicosis tardio Late At-Risk of Psychosis State (LRPS), determinado por los síntomas psicóticos atenuados del criterio UHR (APS y BLIPS) y 3. Estado de psicosis temprana que implica la presencia de un síntoma psicótico franco durante más de una semana. Naturalmente, las medidas preventivas se centran en ambos estados de riesgo, con la diferencia de que en el ERPS se focalizan más en intervenciones psicológicas de tipo cognitivo-conductual, mientras que en el LRPS también se podría justificar la inclusión de tratamiento psicofarmacológico. ${ }^{37}$

Aunque en un inicio los criterios de riesgo según SB y UHR fueron desarrollados de forma independiente, los investigadores están combinando cada vez más ambas aproximaciones, ${ }^{23}$ como ocurre en el Outreach and Support in South London (OASIS), ${ }^{40}$ donde se mejora la sensibilidad de la entrevista CAARMS introduciendo el subgrupo de SB cognitivos de la SPI-A. Esta estratagema también está siendo llevada a cabo por nuestro grupo de investigación en la Fundació Sanitària Sant Pere Claver. ${ }^{41,42}$ Uno de los ejemplos más importantes en cuanto envergadura se refiere es el European Prediction of Psychosis Study (EPOS), ${ }^{43}$ un estudio multicéntrico formado por una muestra inicial de 245 sujetos con riesgo de psicosis definidos según criterios UHR y COGDIS. Se encontró una transición a psicosis al cabo de 18 meses del 19\% (37 sujetos) y un solapamiento entre criterios de riesgo del 59.6\%, siendo la combinación de ambos criterios la estrategia predictiva más sensible. De forma similar, dos estudios igualmente importantes enmarcados en el Ducth Prediction of Psychosis Study (DUPS) ${ }^{27,44}$ también hallaron un solapamiento similar entre criterios UHR y COGDIS; sin embargo, en el estudio de Ziermans et al., ${ }^{27}$ basado en una muestra de 72 pacientes entre 12 y 18 años definida por los mismos criterios de inclusión que en el estudio EPOS, no se encontró que el criterio de 
riesgo COGDIS aportara un valor discriminativo suplementario de transición a psicosis. Este resultado equívoco sobre la validez del criterio COGDIS reafirma la necesidad de incrementar las investigaciones en este tipo de población y en el uso de instrumentos adaptados a ella, como la SPI-CY.

Si bien se han identificado muchos SB altamente específicos de psicosis y combinados de forma exitosa con otros criterios de riesgo, éstos tienen una naturaleza más bien inespecífica, no característica, pudiendo ser encontrados también en otros trastornos del espectro psicótico-afectivo. Este aspecto, más el hecho de que las alteraciones afectivas, como la depresión, sean altamente prevalentes, incluso definitorias, de muchos estados prodrómicos psicóticos, ${ }^{45}$ legitima la duda acerca del poder diagnóstico diferencial de los SB. En este sentido, un estudio encontró que el grupo de sujetos con estado potencialmente prodrómico y el grupo con esquizofrenia diagnosticada no diferían en cuanto a nivel de SB, pero sí que ambos mostraban niveles mucho más altos de SB que el grupo de sujetos con depresión no psicótica, especialmente en las subescalas de la SPI-A alteraciones cognitivas y alteraciones en la vivencia del Yo y del entorno. ${ }^{13}$ Anteriormente, otro estudio focalizado en evaluar las anomalías del self mediante la BSABS en pacientes con esquizofrenia residual y trastorno bipolar ${ }^{46}$ encontró que, comparativamente con el grupo bipolar, los sujetos con diagnóstico de esquizofrenia presentaban puntuaciones más altas en perplejidad (pérdida de significado inmediato), alteraciones perceptivas, alteraciones de la autoconciencia y alteraciones cognitivas.

Atendiendo a los resultados antes expuestos, además del grupo de SB altamente predictivos (mayoritariamente síntomas de tipo cognitivo), también existirían numerosos fenómenos de conciencia alterada o anomalías del self muy importantes y característicos de los trastornos del espectro esquizofrénico. En esta línea, investigaciones posteriores que han evaluado muestras de sujetos con trastornos del espectro psicótico mediante la BSABS han confirmado que los diagnósticos de esquizofrenia y esquizotipia predicen altos niveles de anomalías del self, y que la presencia de dichas anomalías puede diferenciar a aquellos sujetos que pertenezcan al espectro psicótico de los que no. ${ }^{47}$ En el estudio longitudinal de cinco años Copenhagen Prodromal Study de Parnas et $a .^{48}$ se corroboró que altos niveles de perplejidad y anomalías del self (como despersonalización, alteraciones de la conciencia y acción o alteraciones de la percepción corporal) podían ser valiosos indicadores diagnósticos de trastornos del espectro.

Es por ello que en los últimos años, y de forma similar a lo ocurrido con los SB, se han llevado a cabo importantes esfuerzos en proporcionar apoyo empírico-predictivo a estas anomalías sutiles o mínimas del flujo normal de la conciencia. Una contribución muy relevante fue el desarrollo de la entrevista semiestructurada Examination of Anomalous Self-Experience (EASE). ${ }^{49}$ Aunque esta entrevista se solapa en muchas descripciones de síntomas con la BSABS (p. ej. en los dominios de las alteraciones cognitivas o cenestesias), la
EASE se focaliza de forma más específica en captar las alteraciones del self en vez de cubrir todas las potenciales AES. ${ }^{49, *}$

Recientemente, y por primera vez, se ha investigado prospectivamente mediante la EASE una muestra de sujetos UHR adultos durante 569 días (49 sujetos de riesgo y 52 controles) para verificar el poder predictivo de las anomalías del self..$^{50}$ Se encontró que las puntuaciones en anomalías del self eran más altas en aquellos casos diagnosticados con algún trastorno del espectro esquizofrénico, se encontró también que la puntuación total de la EASE predecía de forma significativa transición a psicosis, y que de las cinco dimensiones de la EASE, dos resultaron ser significativamente más predictivas: cognición y corriente de conciencia, autoconciencia e implicación en el mundo (presencia). En referencia a muestras de sujetos menores de edad, un estudio que evaluó por primera vez mediante la EASE y la Structural Interview for Psychosis Syndromes (SIPS) a una muestra de 87 pacientes adolescentes (14 a 18 años), ${ }^{51}$ con problemas emocionales y conductuales encontró que las anomalías del self eran bastante prevalentes entre adolescentes con alteraciones clínicas, aunque de forma sorprendente con una tasa considerablemente menor que los síntomas psicóticos subclínicos medidos con la SIPS. Se constató además un solapamiento moderado entre las alteraciones del self y los síntomas prodrómicos, sugiriéndose que ambos grupos de síntomas podrían representar dos marcadores distintos pero relacionados de vulnerabilidad clínica de psicosis. Este último resultado va en la línea de la demostrada complementariedad entre los criterios de riesgo según SB y UHR en muestras de adultos, pero no desentraña el posible rol generativo de las anomalías del self en los síntomas psicóticos. Pese a lo interesante de estos resultados, se precisa de más investigaciones prospectivas que sigan apuntalando la validez empírica de esta aproximación fenomenológica y que aborden el diseño de unos criterios de riesgo que maximicen su operatividad clínica.

\section{CONCLUSIÓN}

El modelo de los SB, y por extensión la aproximación de las anomalías de la experiencia subjetiva (AES), se posicionan como alternativa fenomenológica a los modelos clínicos basados en los síntomas cardinales de las psicosis, y como complemento altamente válido en las estrategias de detección e intervención temprana de psicosis. Las investigaciones han demostrado mediante rigurosos métodos empíricos que un subgrupo de SB de tipo cognitivo y perceptivo tienen al menos la misma capacidad predictiva de primer episodio de psicosis que los criterios UHR, y que la combinación de ambas estrategias incrementa mutuamente la precisión predictiva de los dos tipos de criterios. Asimismo, la evaluación

* Para una revisión en castellano de esta entrevista y sus escalas, véase PérezÁlvarez et al. ${ }^{11}$ 
prospectiva de las anomalías del self también se está erigiendo como un prometedor campo de investigación empírico que enriquecerá y perfilará el síndrome de riesgo de psicosis, definido hasta ahora mediante los síntomas psicóticos subclínicos (UHR) y en algunos casos mediante algunos SB muy predictivos (COPER/COGDIS). Por todo ello, las AES y los instrumentos que las evalúan son ineludibles para todo aquel clínico interesado en la investigación, comprensión y tratamiento de la sutil pero determinante emergencia de los trastornos del espectro psicótico.

\section{Financiamiento}

Ninguno.

\section{Conflicto de intereses}

Los autores declararon no tener conflicto de intereses.

\section{Agradecimientos}

Neus Barrantes-Vidal agradece al Ministerio de Economía y Competitividad (PSI2011-30321-C02-01), a la Fundació La Marató de TV3 (091110), a la Generalitat de Catalunya (2009SGR672) y a la Institució Catalana de Recerca i Estudis Avanşats (ICREA, Programa Acadèmia) el apoyo brindado a las investigaciones que realiza en esta materia.

\section{REFERENCIAS}

1. Parnas J, Handest, P. Phenomenology of anomalous self-experience in early schizophrenia. Compr Psychiatry 2003;44(2):121-134.

2. Peralta V, Cuesta MJ. Subjective experiences in schizophrenia: A critical review. Compr Psychiatry 1994;35(3):198-204.

3. Sass L, Parnas J. Phenomenology of self-disturbances in schizophrenia: Some research findings and directions. Philos Psychiatr Psychol 2001;8(4):347-356.

4. Sass L. Self-disturbance and schizophrenia: Structure, specificity, pathogenesis (Current issues, New directions). Schizophr Res 2013;S0920-9964(13):272-277.

5. Lysaker PH, Lysaker JT. Schizophrenia and alterations in self-experience: A comparison of 6 perspectives. Schizophr Bull 2010;36(2):331-340.

6. Gross G. The "basic" symptoms of schizophrenia. Br J Psychiatry 1989;155(suppl):21-25.

7. Gross G, Huber G. The history of the basic symptom concept. Acta Clin Croat 2010;49(2):47-59.

8. Huber G, Gross G. The concept of basic symptoms in schizophrenic and schizoaffective psychoses. Recenti Prog Med 1989;80(12):646-652.

9. Schultze-Lutter F, Klosterkötter J, Picker H, Steinmeyer EM et al. Predicting first-episode psychosis by Basic Symptom Criteria. J Clin Neuropsychiatry 2007;4(1):11-22.

10. Schultze-Lutter F. Subjective Symptoms of Schizophrenia in Research and the Clinic: The Basic Symptom Concept. Schizophr Bull 2009;35(1):5-8.

11. Pérez-Alvarez M, García-Montes J, Sass L. La hora de la fenomenología en la esquizofrenia. Clínica Salud 2010;21(3):221-233.

12. Jimeno-Bulnes $\mathbf{N}$, Jimeno-Valdés $A$, Vargas ML. El síndrome psicótico y el Inventario de Frankfurt: conceptos y resultados. Barcelona: Springer-Verlag Ibérica; 1996.

13. Schultze-Lutter F, Ruhrmann S, Picker H, von Reventlow et al. Basic symptoms in early psychotic and depressive disorders. Br J Psychiatry 2007;51:31-37.
14. Gross G. The onset of schizophrenia. Schizophr Res 1997;28:187-198.

15. Klosterkötter J, Hellmich M, Steinmeyer EM, Schultze-Lutter F. Diagnosing schizophrenia in the initial prodromal phase. Arch Gen Psychiatry 2001;58(2):158-164.

16. Gross G, Huber G, Klosterkötter J, Linz M. Bonner Skala für die Beurteilung von Basis-symptomen -Bonn Scale for the Assessment of Basic Symptom. Berlin: Springer; 1987.

17. Klosterkötter J, Gross G, Huber G, Wieneke A et al. Evaluation of the Bonn Scale for the assessment of basic symptoms -BSABS- as an instrument for the assessment of schizophrenia proneness: a review of recent findings. Neurol Psychiatr Brain Res 1997;5:137-150.

18. Schultze-Lutter F, Ruhrmann S, Klosterkötter J. Can schizophrenia be predicted phenomenologically? En: Johannessen JO, Martindale B, Cullberg J (eds.). Evolving psychosis. Different stages, different treatments. Londres, Nueva York: Routledge: 2006; pp.104-123.

19. Schultze-Lutter F, Addington J, Ruhrmann S, Klosterkötter J. Schizophrenia Proneness Instrument -Adult version (SPI-A). Rome: Giovanni Fioriti; 2007.

20. Schultze-Lutter F, Koch E. Schizophrenia Proneness Instrument Child and Youth version (SPI-CY). Rome: Giovanni Fioriti; 2010.

21. Schultze-Lutter F, Ruhrmann S, Klosterkötter J. Early detection of psychosis- establishing a service for persons at risk. Eur Psychiatry 2009;24(1):1-10.

22. Kay SR, Fiszbein A, Opler LA. The Positive and Negative Syndrome Scale (PANSS) for schizophrenia. Schizophr Bull 1987;13:261-276.

23. Schultze-Lutter F, Ruhrmann S, Fusar-Poli P, Bechdolf A et al. Basic symptoms and the prediction of first-episode psychosis. Curr Pharm Des 2012;18(4):351-357.

24. Schultze-Lutter F, Steinmeyer EM, Ruhrmann S, Klosterkötter J. The dimensional structure of self-reported "prodromal" disturbances in Schizophrenia. J Clin Neuropsychiatry 2008;5(3):140-150.

25. Schimmelmann BG, Walger P, Schultze-Lutter F. The significance of At-Risk Symptoms for Psychosis in Children and Adolescents. Can J Psychiatry 2013;58(1):32-40.

26. Cornblatt BA, Lencz T, Smith CW, Olsen R et al. Can antidepressants be used to treat the schizophrenia prodrome? Results of a prospective, naturalistic treatment study of adolescents. J Clin Psychiatry 2007; 68(4):546-557.

27. Ziermans T, Schothorst P, Sprong M, van Engeland H. Transition and remission in adolescents at ultra-high risk for psychosis. Schizophr Res 2011;126:58-64.

28. Meng H, Schimmelmann BG, Koch E, Bailey B et al. Basic symptoms in the general population and in psychotic and non-psychotic psychiatric adolescents. Schizophr Res 2013;111(1-3):32-38.

29. Schultze-Lutter F, Schimmelmann BG, Koch E. The dimensional structure of prodromal symptoms in early-onset psychosis: Indication of special needs in the early detection of younger adolescents. Schizophr Res 2010;117:2-3.

30. Fux L, Walger P, Schimmelmann BG, Schultze-Lutter F. The Schizophrenia proneness instrument, child and youth version (SPI-CY): Practicability and discriminative validity. Schizophr Res 2013;146:6978.

31. Koch E, Schultze-Lutter F, Schimmelmann BG, Resch F. On the importance and detection of prodromal symptoms from the perspective of child and adolescent psychiatry. J Clin Neuropsychiatry 2010;2:3848.

32. McGorry PD, Nelson B, Amminger GP, Francey SM et al. Intervention in individuals at ultra-high risk for psychosis: a review and future directions. J Clin Psychiatry 2009;70(9):1206-1212.

33. Yung AR, McGorry PD, McFarlane CA, Jackson, HJ et al. "Monitoring and care of young people at incipient risk of psychosis". Schizophr Bull 1996;22(2):283-303.

34. Cornblatt BA, Lencz T, Smith CW, Correll CU et al. The schizophrenia prodrome revisited: a neurodevelopmental perspective. Schizophr Bull 2003;29(4):633-651. 
35. Yung AR, Yuen HP, McGorry PD, Phillips LJ et al. Mapping the onset of psychosis: the Comprehensive Assessment of At-Risk Mental States. Aust N Z J Psychiatry 2005;39(11-12):964-971.

36. McGlashan T, Walsh B, Woods S. The Psychosis-Risk Syndrome. Handbook for diagnosis and follow-up. Oxford: University Press; 2010.

37. Klosterkotter J, Schultze-Lutter F, Bechdolf A, Ruhrmann S. Prediction and prevention of schizophrenia: what has been achieved and where to go next? World Psychiatry 2011;10(3):165-174.

38. Schultze-Lutter F, Ruhrmann S, Berning J, Maier W et al. Basic symptoms and ultrahigh risk criteria: Symptom development in the initial prodromal state. Schizophr Bull 2008;36(1):182-191.

39. Simon AE, Dvorsky DN, Boesch J, Roth B et al. Defining subjects at risk for psychosis: A comparison of two approaches. Schizophr Res 2006;81:83-90.

40. Fusar-Poli P, Borgwardt S, Valmaggia L. Heterogeneity in the assessment of the at-risk mental state for psychosis. Psychiatr Serv 2008;59(7):813.

41. Domínguez-Martínez T, Blanqué JM, Codina J, Montoro M et al. Rationale and state of the art in early detection and intervention in psychosis. Salud Mental 2011;34(4):341-350.

42. Domínguez-Martínez T, Vainer E, Massanet MA, Torices I et al. The need-adapted integrated treatment in Sant Pere Claver-Early Psychosis Program (SPC-EPP) in Barcelona, Spain. Salud Mental 2011;34(6):517-524.

43. Ruhrmann S, Schultze-Lutter F, Salokangas RKR, Heinimaa M et al. Prediction of psychosis in adolescents and young adults at high risk: results from the prospective European prediction of psychosis study. Arch Gen Psychiatry 2010;67 (3):241-251.

44. Velthorst E, Nieman DH, Becker HE, van de Fliert et al. Baseline differences in clinical symptomatology between ultra high risk subjects with and without a transition to psychosis. Schizophr Res 2009;109(1-3):60-65.

45. Häfner H, Löffler W, Maurer K, Hambrecht M et al. Depressión, negative symptoms, social stagnation and social decline in the early course of schizophrenia. Acta Psychiatr Scand 1999;100(2):105-118.

46. Parnas J, Handest P, Saebye D, Janson L. Anomalies of subjective experience in Schizophrenia and psychotic bipolar illness. Acta Psychiatr Scand 2003;108:126-133.

47. Raballo A, Sæbye D, Parnas J. Looking at the schizophrenia spectrum through the prism of self-disorders: an empirical study. Schizophr Bull 2011;37(2):344-351.

48. Parnas J, Raballo A, Handest P, Janson L et al. Self-experience in the early phases of schizophrenia: 5-year follow-up of the Copenhagen Prodromal Study. World Psychiatry 2011;10(3):200-204.

49. Parnas J, Møller P, Kircher T, Thalbitzer J et al. EASE: Examination of anomalous self-experience. Psychopathology 2005;38(5):236-258.

50. Nelson B, Thompson A, Yung R. Basic self-disturbance predicts psychosis onset in the ultra high risk for psychosis "prodromal" population. Schizophr Bull 2012;38 (6):1277-1287.

51. Koren D, Reznik N, Adres M, Scheyer R et al. Disturbances of basic self and prodromal symptoms among non-psychotic help-seeking adolescents. Psychol Med 2013;43(7):1365-3176. 